\title{
Sequential order of swing phase initiation in baseball
}

\author{
Ethan Stewart $^{\mathrm{a}, *}$, Megan Stewart ${ }^{\mathrm{b}}$, Jeffrey Simpson ${ }^{\mathrm{c}}$, Adam Knight $^{\mathrm{a}}$, Harish Chander $^{\mathrm{a}}$ \\ and Robert Shapiro ${ }^{\mathrm{d}}$ \\ ${ }^{a}$ Department of Kinesiology, Mississippi State University, Mississippi State, MS, USA \\ ${ }^{\mathrm{b}}$ The American Sports Medicine Institute, Birmingham, AL, USA \\ ${ }^{\mathrm{c}}$ Department of Movement Sciences and Health, University of West Florida, Pensacola, FL, USA \\ ${ }^{\mathrm{d}}$ Department of Kinesiology and Health Promotion, University of Kentucky, Lexington, KY, USA
}

\begin{abstract}
In order to successfully hit a baseball, hitters must utilize a series of preparatory movements (swing phases) which include shifting their body weight, stepping, landing, and swinging. The purpose of this study was to examine the differences between start times for swing phases (shifting, stepping, landing, and swinging)for currently active baseball players. Participants $(n=12)$ were all current collegiate baseball athletes. Retroreflective markers, surface electromyography (EMG) and two force platforms were utilized to complete a swing analysis. Each participant completed five swinging trials off a tee. All dependent variables were compared using a repeated measures $1 \times 4$ ANOVA with LSD post hoc comparison $(p<0.05)$ if necessary. The results demonstrated that the participants started the swing phases in a statistically significant sequence of shifting, stepping, landing, and swinging. The ability of the athletes to start the swing phases in this sequential order may be advantageous to regulate spatial parameters of their swing and provide more time to generate power. These results allow for coaches to better understand how to instruct their athletes to be successful at the plate.
\end{abstract}

Keywords: Biomechanics, kinematics, sport performance, coaching, hitting

\section{Introduction}

Baseball is played around the world by athletes of varying ability, which leads to an inconsistency of skill level within the game and across leagues. Hitting a baseball has been defined as one of the most difficult skills in sports, thus, understanding how variations in skill level can influence the success and proper progression of hitting is of great concern to athletes and coaches (DeRenne, 2007). While previous studies have investigated the spatial characteristics (joint angles) of successful baseball swings and their relation to skill level (Escamilla et al., 2009a, Inkster et al., 2011, Dowling and Fleisig, 2016), only two

\footnotetext{
*Corresponding author: Ethan Stewart, PO Box 6186, Department of Kinesiology, Mississippi State, MS 39762 USA. Tel.: +1 502480 6194; E-mail: ems664@msstate.edu.
}

studies have identified the temporal characteristics (swing phases) (Escamilla et al., 2009a, Nakata et al., 2013). Thus, identifying temporal swing characteristics that could potentially enhance performance when hitting a baseball is also an important characteristic to assess when working to develop a player's skill.

In order to successfully hit a baseball, hitters must utilize a series of movements including shifting their body weight to the back foot (loading), stepping (striding) with the lead foot, landing, swinging (taking the hands to the ball), and following through (Nakata et al., 2013). When analyzing hitters in different age groups, adult (college and professional) baseball hitters have been found to spend a significantly longer amount of time between the stepping and landing phases of the swing compared to youth hitters (Escamilla et al., 2009a). Adult hitters also 
take a significantly longer time to complete the entire swing than youth hitters (Escamilla et al., 2009a). In order to compensate for the increased pitch velocity that an adult hitter would see in competition, the swing is required to start significantly earlier if it takes longer to complete. Starting the swing earlier will allow the hitter to complete the swing despite decreasing the amount of response time they have to make contact once the ball leaves the pitcher's hand. As baseball players progress through competition levels, their swing must also progress to retain the probability of being successful when hitting.

In an investigation between skilled hitters who had collegiate or higher level experience and novice hitters, specific swing phase differences were identified (Nakata et al., 2013) The skilled group exhibited significantly earlier initiation for the shifting, stepping, and landing phases, followed by significantly later initiation of the swinging phase when compared to the novice group (Nakata et al., 2013). This study provided insight into the skill of hitting a baseball, but the differences between the groups leave questions about how athletes must properly progress through their swing phases to be successful at the plate (Nakata et al., 2013). It is also noted that the study completed by Nakata et al. (2013) did not use currently active baseball players and only required them to be competing in baseball once a week after graduating college. This study also identified the stepping and landing phases using video (Nakata et al., 2013). Therefore, the purpose of this study was to examine the difference between start times for the swing phases (shifting, landing, stepping, and swinging) for currently active collegiate baseball athletes utilizing motion capture, surface electromyography, and force platforms. The authors hypothesized that the athletes would exhibit a forward progression through the swing phases from shifting, landing, stepping, and swinging. This study will allow coaches to identify the correct order of the swing phases to help facilitate success at the plate.

\section{Materials and methods}

\subsection{Participants}

Twelve male baseball players (age: $23.67 \pm$ 3.39 years, height: $1.76 \pm 0.05 \mathrm{~m}$, weight: $86.67 \pm$ $7.97 \mathrm{~kg}$ ) that were currently partaking in competitive collegiate baseball participated in this study. All testing received approval from the Institutional
Review Board (IRB 17-0183-P4S) for the use of human participants and occurred immediately outside of the collegiate baseball season in the summer. An approved IRB consent form that defined all risk and procedures of the study was read and signed by the participant prior to the initiation of data collection procedures.

\subsection{Protocol}

All participants were instructed to avoid any strenuous activity for the 24 hours prior to their data collection session to minimize the risk of fatigue. Each participant was given standardized athletic footwear (Nike, Beaverton, OR) to wear for the duration of their data collection session and were fitted with two retroreflective markers on the distal end of the third metacarpal bone of the left hand to define the proximal end of the bat. The baseball was fitted with a single retroreflective marker and the bat was fitted with two retroreflective markers at the midpoint and distal end to track bat and ball movement. Retroreflective markers were used only to track the motion of the bat, ball, and lead hand. One surface electromyography electrode was placed on the trail leg rectus femoris to determine the start of the shifting phase (see Swing Analysis). Placement of the electrode was defined based on SENIAM guidelines (Merletti and Hermens, 2000). Before placement of the electrode, the skin was shaved, abraded lightly with sandpaper and cleaned with alcohol to avoid impedance between the skin and electrode. A standardized warm-up of ten swings was given to each participant after they were given time to warm-up as they normally would before hitting (Escamilla et al., 2009b, Escamilla et al., 2009a). Participants were asked to perform the swing as they normally would. In order to accurately obtain force plate data for each leg, each participant placed one foot on two separate force plates such that both feet would remain completely on a single force plate when in contact with the ground, for the entirety of the swing. Each participant performed five swing trials off a tee while their swing data were recorded.

The swing analysis was completed using two force plates (Bertec, Columbus, $\mathrm{OH}$ ) recording at $1000 \mathrm{~Hz}$, 6 Eagle and 4 Raptor Motion Analysis cameras (Motion Analysis Corp, Santa Rosa, CA) recording at $200 \mathrm{~Hz}$, and surface electromyography (Delsys, Natick, MA) recording at $1000 \mathrm{~Hz}$. A static image of each participant was captured to define the bat markers. Each participant was allotted 10 warm-up swings to become acclimated to the laboratory setting followed 
by five swing trials during which marker trajectory, force, and sEMG data were collected. If a trial was performed in which the hitter did not completely land with his lead foot entirely on the force platform the swing was completed again.

\subsection{Swing analysis}

The swing was broken down into seven phases and defined based on previous literature (Nakata et al., 2013): waiting, shifting, stepping, landing, swinging, impact and follow-through. The beginning of the waiting phase was defined as the time before the shifting phase. The beginning of the shifting phase was defined as the muscle onset of the trail leg rectus femoris by examining the sEMG data and this phase ended at the beginning of the stepping phase. The stepping phase began when the front foot was lifted and the force platform reading went below $10 \mathrm{~N}$. The landing phase began when the front foot was replaced on the force platform and the force exceeded $10 \mathrm{~N}$. The beginning of the swinging phase marked the end of the landing phase and was defined as the point in which the bat started to move downward toward the ball. The start of the swinging phase was defined by the marker trajectory data of the lead hand. The swinging phase ended at ball impact/contact and the follow-through phase began and continued for half of a second after contact. All swing phases were defined based on previous literature from Nakata et al. (Nakata et al., 2013). For the purpose of this study, only the shifting, stepping, landing, and swinging phase were compared.

\subsection{Data processing}

Marker trajectory, force platform, and sEMG data to calculate all variables of interest were collected simultaneously using Cortex software (Motion Analysis Corp., Santa Rosa, CA). Data processing including filtering of marker trajectory data, force platform output to determine the start of the stepping and landing phase was conducted using Visual 3D software (C-Motion Inc., Germantown, MD). Raw marker trajectory data were filtered using a fourth order low-pass Butterworth filter with a cut-off frequency of $13 \mathrm{~Hz}$. Frequency cut-off was determined using a frequency analysis graph. Onset timing for the trail leg rectus femoris was determined using Noraxon myoRESEARCH 3.10 (Noraxon U.S.A. Inc., Scottsdale, AZ) with onset defined as three
Table 1

ANOVA test results for the four phases of the baseball swing

\begin{tabular}{lcccc}
\hline Variable & F-value & $p$-value & $\eta_{p}^{2}$ & Power \\
\hline Swing Phases & $\mathrm{F}(1,11)=93.977$ & $<0.001$ & 0.895 & 1.00 \\
\hline
\end{tabular}

standard deviations (Bolgla et al., 2010) above the baseline activity of the stance (prior to initiation of the swing) to define the start of the shifting phase(Nakata et al., 2013). Raw sEMG signal were filtered using a finite impulse response with 101 points using Hamming window and smoothed with a root mean-square moving average of $10 \mathrm{~ms}$ per window. GRF data was not filtered in order to most accurately identify when the lead foot was lifted and reapplied to the ground. Initiation of the swing phase was defined as milliseconds (ms) before ball contact. Data were then averaged for the athletes to compare the potential differences between the start time for swing phases.

\subsection{Statistical analysis}

Descriptive and dependent variables are reported as mean \pm standard deviation (SD). Differences in the start times for swing phases were all compared using a repeated measures $1 \times 4$ ANOVA followed by an LSD comparison of the means to determine which phases were significantly different from one another. All statistical procedures were performed using Statistical Package for Social Sciences 24 (SPSS) software (SPSS Inc., Chicago, IL) with a significance level of $p<0.05$.

\section{Results}

\subsection{Swing phases}

The F-value, $p$-value, partial eta squared, and power from the Repeated Measure $1 \times 4$ ANOVA can be found in Table 1. Mean values, standard deviation, and statistical significance as determined from LSD comparisons for start times of each swing phase can be found in Table 2. All data are referenced in milliseconds (ms) to contact, with contact representing zero ms. A graphical representation of the swing phase start times can be found in Fig. 1. Results show a statistically significant sequential order of the start times of swing phases. 
Table 2

Start time (Mean $\pm \mathrm{SD}$ ), for the four phases of the baseball swing

\begin{tabular}{lcccc}
\hline Phases & Shifting & Stepping & Landing & Swinging \\
\hline $\begin{array}{l}\text { Timing (ms before } \\
\text { contact) }\end{array}$ & $-766.39 \pm 181.28^{*}$ & $-544.17 \pm 91.03^{\#}$ & $-296.67 \pm 60.01^{\beta}$ & $-214.86 \pm 44.05$ \\
\hline
\end{tabular}

*Denotes significant difference between the shifting phase and the stepping, landing, and swinging phases. ${ }^{*}$ Denotes significant difference between the stepping phase and the landing and swinging phases. ${ }^{\beta}$ Denotes significant difference between the landing phase and the swinging phase.

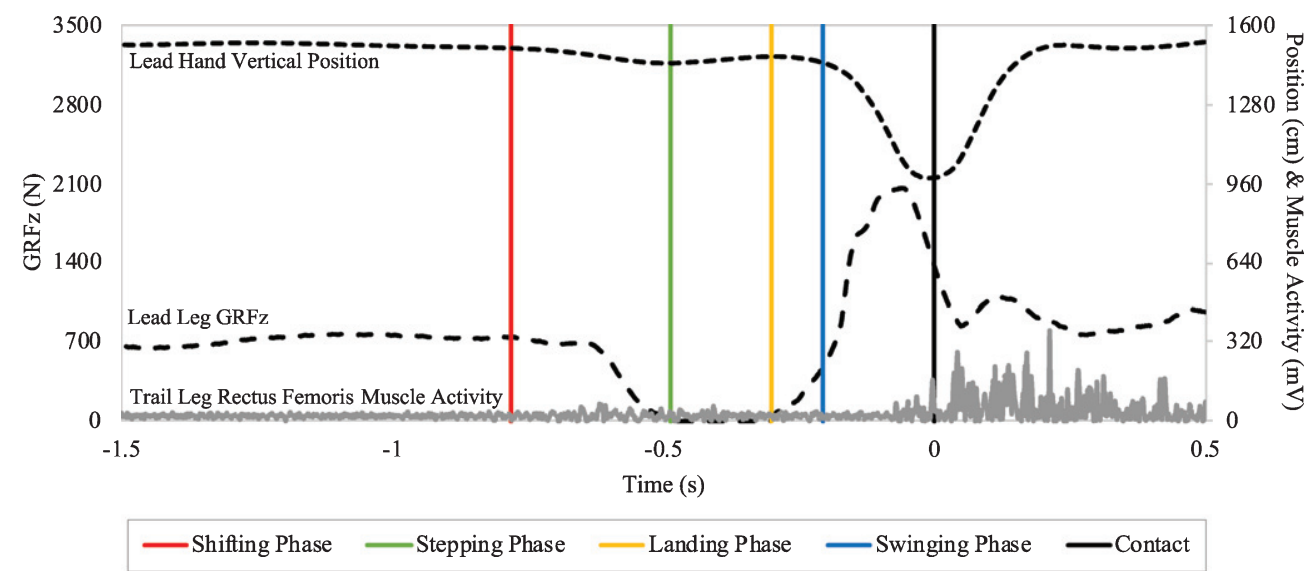

Fig. 1. Start times of swing phases as defined by the onset of the trail leg rectus femoris, lead leg GRFz, and lead hand vertical position.

\section{Discussion}

The purpose of this study was to examine the difference between start times of swing phases (shifting, landing, stepping, and swinging) for currently competitive collegiate baseball players. Based on previous research (Nakata et al., 2013, Escamilla et al., 2009a, Dowling and Fleisig, 2016, Inkster et al., 2011), it was hypothesized that the athletes would start the shifting, stepping, landing, and contact phases in a sequential order. Agreeing with our hypothesis and previous research (Escamilla et al., 2009a, Nakata et al., 2013), the swing phases had a statistically significant sequential order. The significant differences seen in the start times of these phases suggests that starting these phases in sequential order is vital to be successful at hitting a baseball. Starting the shifting, stepping, landing, and swinging phases in order could be the key for hitters to be able to develop the power needed to hit the ball by taking better advantage of the kinetic chain (Race, 1961).

Starting the shifting phase significantly earlier than the other swing phases allows the hitter a greater amount of time to prepare for the swing. A greater duration of time allotment for developing power in the swing would allow the hitter greater time to utilize the kinetic chain of the swing to generate a higher bat velocity. Statistical significance for starting the swinging phase significantly later than the other phases allows the hitter to have significantly more time to see the ball. When a hitter is able to see the baseball, or in this case, see the baseball for a greater period of time, it has been found that they will more successfully make bat-ball contact (Witt and Proffitt, 2005). It has also been shown in previous research that higher level hitters are better able to make adjustments to changes in pitch type than lower level hitters in order to successfully hit a baseball (Gray, 2002). The decreased time needed to successfully hit the baseball found in this study could give insight as to why the differences may be seen in the higher level or more skilled hitters.

Previous research determined that novice athletes showed significantly different swing phase orders than skilled athletes (Nakata et al., 2013). The purpose of the current study was to further investigate the sequential order of the swing phases for athletes currently competing at the collegiate level. The results of this study show similarities to previous research in the order that swing phases were completed (Escamilla et al., 2009a, Nakata et al., 2013). The sequential timing of baseball pitching has been shown to be of utmost importance to the success of the pitcher and has been used to investigate success of the athlete 
(Urbin et al., 2013, Fleisig et al., 2009, Werner et al., 1993, Escamilla et al., 1998). The definition of the proper progression of the phases of the baseball pitch has allowed further investigation into kinematic and kinetic properties that could affect the success of the pitcher. The results of the current study show a statistically significant sequential order of the swing phases for baseball athletes. These findings allow for coaches to better understand how to instruct their athletes to succeed at the plate, and for further research to investigate how kinematic and kinetic properties of the swing relate to the swing phases.

There are several limitations that should be noted in this study. sEMG sensors and marker placement error are always present. To control for this, a single investigator applied all sensors and markers to avoid inter-tester variability. Errors involving skin movement artifact should also be noted as markers were placed directly on skin and do not truly represent the bony landmarks. Participant's adherence to directions could also limit this study. All participants were asked to avoid any strenuous activity for the twenty-four hours prior to data collection to help avoid muscle fatigue. Non-adherence to this request could lead to misinterpretations of muscle onset during the swing. It was assumed that all participants avoided strenuous activity. It was assumed that all participants were giving full effort when performing all tasks for this study.

Future research should focus on locating possible differences between the kinematic and kinetic properties of baseball hitters during these specific sewing phases. Further research should also investigate how the swing phases differ between hitters of varying skill or competition level. While this study gives insight as to differences within the collegiately active baseball hitters, it may be beneficial to examine the differences in timing of the swing phases for baseball hitters playing on different competition levels. Information between competition levels would give valuable insight as to the progression needed to stay successful when transitioning to a higher competition level.

\subsection{Practical applications}

The statistical differences that are identified between the swing phases in collegiate hitters found in this study can be used by coaches to help better understand the progression that athletes should demonstrate. Utilizing the data found for collegiately active athletes, coaches can implement training and teaching techniques to guide their hitters toward the proper progression of the swing phases to be successful. While coaching athletes on millisecond differences in their swing could prove difficult, it is believed that these parameters could help coaches instruct their athletes on how to adjust the start of a certain phase based on how they perform. With the knowledge of how the athlete compares to hitters at the collegiate level, coaches could be better situated to coach them and determine if the athletes are starting their swing phases in the shown significant sequential order.

\subsection{Conclusion}

Significant differences in the start times for the shifting, stepping, landing and swinging phase were reported. These differences could contribute to the success of making contact while trying to hit a baseball. By starting the swing phases in a sequential order, the hitters have more time to prepare for the swing by regulating spatial parameters and allowing more time to generate power and successfully make bat contact with the ball. This increased time to generate power in the swing could lead to higher bat velocity and overall skill level of the athlete.

\section{References}

Bolgla, L. A., Malone, T. R., Umberger, B. R. and Uhl, T. L. 2010. Reliability of electromyographic methods used for assessing hip and knee neuromuscular activity in females diagnosed with patellofemoral pain syndrome. Journal of Electromyography and Kinesiology, 20, 142-147.

DeRenne, C. 2007. The scientific approach to hitting: Research explors the most difficult skill in sport, University Readers.

Dowling, B. and Fleisig, G. S. 2016. Kinematic comparison of baseball batting off of a tee among various competition levels. Sports Biomech, 15, 255-269.

Escamilla, R. F., Fleisig, G. S., Barrentine, S. W., Zheng, N. and Andrews, J. R. 1998. Kinematic comparisons of throwing different types of baseball pitches. Journal of Applied Biomechanics, 14, 1-23.

Escamilla, R. F., Fleisig, G. S., DeRenne, C., Taylor, M. K., Moorman, C. T., 3RD, Imamura, R., Barakatt, E. and Andrews, J. R. 2009a. A comparison of age level on baseball hitting kinematics. J Appl Biomech, 25, 210-218.

Escamilla, R. F., Fleisig, G. S., DeRenne, C., Taylor, M. K., Moorman, C. T., 3RD, Imamura, R., Barakatt, E. and Andrews, J. R. 2009b. Effects of bat grip on baseball hitting kinematics. J Appl Biomech, 25, 203-209.

Fleisig, G., Chu, Y., Weber, A. and Andrews, J. 2009. Variability in baseball pitching biomechanics among various levels of competition. Sports Biomechanics, 8, 10-21. 
Gray, R. 2002. "Markov at the bat": a model of cognitive processing in baseball batters. Psychol Sci, 13, 542-547.

Inkster, B., Murphy, A., Bower, R. and Watsford, M. 2011. Differences in the Kinematics of the Baseball Swing between Hitters of Varying Skill. Medicine and Science in Sports and Exercise, 43, 1050-1054.

Merletti, R. and Hermens, H. 2000. Introduction to the special issue on the SENIAM European Concerted Action. J Electromyogr Kinesiol, 10, 283-286.

Nakata, H., Miura, A., Yoshie, M., Kanosue, K. and Kudo, K. 2013. Electromyographic analysis of lower limbs during baseball batting. J Strength Cond Res, 27, 1179-1187.

Race, D. E. 1961. A cinematographic and mechanical analysis of the external movements involved in hitting a baseball effectively. Research Quarterly. American Association for Health, Physical Education and Recreation, 32, 394-404.

Urbin, M., Fleisig, G. S., Abebe, A. and Andrews, J. R. 2013. Associations between timing in the baseball pitch and shoulder kinetics, elbow kinetics, and ball speed. The American Journal of Sports Medicine, 41, 336-342.

Werner, S. L., Fleisig, G. S., Dillman, C. J. and Andrews, J. R. 1993. Biomechanics of the elbow during baseball pitching. Journal of Orthopaedic \& Sports Physical Therapy, 17, 274-278.

Witt, J. K. and Proffitt, D. R. 2005. See the ball, hit the ball. Psychol Sci, 16, 937-938. 